\title{
Moments of Progressive Type-II Right Censored Order Statistics from a General Class of Doubly Truncated Continuous Distributions
}

\author{
Jagdish Saran* \\ Department of Statistics, University of Delhi \\ Delhi-110007, India \\ jagdish_saran52@yahoo.co.in \\ Narinder Pushkarna \\ Department of Statistics, University of Delhi \\ Delhi-110007, India \\ drpushkarna@hotmail.com
}

Received 6 July 2013

Received 4 April 2014

\begin{abstract}
In this paper we derive some general recurrence relations between moments of progressively Type-II right censored order statistics from a general class of doubly truncated distributions, thus unifying the earlier results in this direction due to several authors.

Keywords: Order Statistics; Progressively Type-II right censored order statistics; Single moments; Product moments; Recurrence relations; Truncated and non-truncated Lomax, Weibull, Weibull-gamma, Weibullexponential, log logistic, exponential, generalized exponential, Rayleigh, generalized Rayleigh, generalized Pareto, linear exponential and Burr distributions.
\end{abstract}

2010 Mathematics Subject Classification: 62G30, 62E10

\section{Introduction}

The scheme of progressive Type-II censoring is of importance in reliability and life-testing experiments. It allows the experimenter to remove units from a life test at various stages during the experiment which may lead to a saving of costs and of time. (see Cohen, 1963 and Sen, 1986). In such a random experiment, a group of $n$ independent and identical experimental units is put on a life test at time zero with continuous, identically distributed failure times $X_{1}, X_{2}, \ldots, X_{n}$. After the $j^{\text {th }}$ failure, a prespecified number $R_{j} \geq 0$ of the $n-j-\sum_{i=0}^{j-1} R_{i}$ remaining (or surviving) units are randomly withdrawn from the experiment, $1 \leq j \leq m, m \leq n, R_{0}=0$. Removed units thus become right censored at the time of failure of other units. This progressive censoring leads to $m$ ordered observed failure times denoted by $X_{1: m: n}^{\left(R_{1}, R_{2}, \ldots, R_{m}\right)}, X_{2: m: n}^{\left(R_{1}, R_{2}, \ldots, R_{m}\right)}, \ldots, X_{m: m: n}^{\left(R_{1}, R_{2}, \ldots, R_{m}\right)}$, and these are called progressively Type-II right censored order statistics of size $m$ from a sample of size $n$ with progressive censoring scheme $\left(R_{1}, R_{2}, \ldots, R_{m}\right)$. Thus, in this type of sampling, $m$ failures are observed, $\sum_{j=1}^{m} R_{j}$ units are progressively censored and $n=m+\sum_{j=1}^{m} R_{j}$ denotes the number of units in the life test. The withdrawal of units may be seen as a model describing drop-outs of units due to failures which

${ }^{*}$ Corresponding author 
have causes other than the specific one under study. In this sense, progressive censoring schemes are applied in clinical trials as well. Here, the drop-outs of patients may be caused by migration, lack of interest or by personal or ethical decisions, and they are regarded as random withdrawals. For a detailed discussion of progressive censoring and the relevant developments in this area, one may refer to Sen (1986), Balakrishnan and Aggarwala (2000) and Aggarwala (2001).

The situation with no censoring corresponds to the special case with $m=n$ and $R_{1}=R_{2}=\ldots=$ $R_{m}=0$, whereas the situation with ordinary Type-II right censoring at a given order statistic corresponds to the special case with $m<n, R_{1}=R_{2}=\ldots=R_{m-1}=0$ and $R_{m}=n-m$.

If the failure times of the $n$ items originally on test are from a doubly truncated continuous population with c.d.f. $F(x)$ and p.d.f. $f(x)$, then the joint p.d.f. of $X_{1: m: n}^{\left(R_{1}, R_{2}, \ldots, R_{m}\right)}, X_{2: m: n}^{\left(R_{1}, R_{2}, \ldots, R_{m}\right)}$, $\ldots, X_{m: m: n}^{\left(R_{1}, R_{2}, \ldots, R_{m}\right)}$ is given by (cf. Balakrishnan and Sandhu (1995) and Saran and Pushkarna (2001))

$$
f_{1,2, \ldots, m: m: n}\left(x_{1}, x_{2}, \ldots, x_{m}\right)=A(n, m-1) \prod_{i=1}^{m} f\left(x_{i}\right)\left[1-F\left(x_{i}\right)\right]^{R_{i}}, \quad Q_{1} \leq x_{1}<x_{2}<\cdots<x_{m} \leq P_{1},
$$

where $A(n, m-1)=n\left(n-R_{1}-1\right)\left(n-R_{1}-R_{2}-2\right) \ldots\left(n-R_{1}-R_{2}-\ldots-R_{m-1}-m+1\right)$.

Here, note that all the factors in $A(n, m-1)$ are positive integers. Also it may be observed that the different factors in $A(n, m-1)$ represent the number of units still on test immediately preceding the first, second, ..., $m^{\text {th }}$ observed failures, respectively. Similarly, for convenience in notation, let us define

$$
A(p, q)=p\left(p-R_{1}-1\right)\left(p-R_{1}-R_{2}-2\right) \ldots\left(p-R_{1}-R_{2}-\cdots-R_{q}-q\right),
$$

for $q=0,1, \ldots, p-1$, with all the factors being positive integers.

Also the quantities $Q_{1}$ and $P_{1}$ in (1.1) are the points of truncation of the p.d.f. $g(x)$ of the untruncated population given by

$$
\int_{-\infty}^{Q_{1}} g(x) d x=Q
$$

and

$$
\int_{P_{1}}^{\infty} g(x) d x=1-P
$$

i.e., $Q$ and $1-P(Q<P)$ are, respectively, the proportions of truncation on the left and right of the p.d.f. $g(x)$, where

$$
f(x)=\frac{g(x)}{P-Q}, \quad Q_{1} \leq x \leq P_{1} .
$$

The quantities $Q$ and $P$ are assumed to be known and $Q_{1}$ and $P_{1}$ are functions of $Q$ and $P$.

By assuming the underlying distribution of failure times as exponential, half logistic, right truncated exponential, doubly truncated exponential, doubly truncated Pareto, doubly truncated power function and doubly truncated Burr, and utilizing the corresponding characterizing differential equation, several authors, viz. Aggarwala and Balakrishnan (1996), Saran and Pande (2012), Balakrishnan and Aggarwala (2000) and Saran and Pushkarna (2001) have derived recurrence relations for single and product moments of the corresponding progressively Type-II right censored order statistics. These recurrence relations will allow one for the recursive computation of these moments for 
all sample sizes and all possible censoring schemes.

In this paper, we derive some general recurrence relations satisfied by the single and product moments of progressively Type-II right censored order statistics from a general class of doubly truncated distributions with p.d.f. $f(x)$ and c.d.f. $F(x)$ satisfying the characterizing differential equation:

$$
\left(\sum_{i=0}^{p} a_{i} x^{i}\right) f(x)=\left(\sum_{j=0}^{q} b_{j} x^{j}\right)(c+\{1-F(x)\}),
$$

or, equivalently,

$$
\left(\sum_{i=0}^{p} a_{i} x^{i}\right) f(x)=\left(\sum_{j=0}^{q} b_{j} x^{j}\right)(d-F(x)),
$$

where $d=1+c$ and $Q_{1} \leq x \leq P_{1}$. Here $p$ and $q$ are integers and $a^{\prime} s, b^{\prime} s$ and $c$ are arbitrary real constants.

Further, for the special case $R_{1}=R_{2}=\ldots=R_{m}=0$, the derived results would reduce to the general recurrence relations for the usual order statistics from the general class of doubly truncated distributions satisfying the characterizing differential equation (1.3) or (1.4).

It is worth mentioning here that several doubly truncated distributions, for example, doubly truncated Lomax, Weibull, Weibull-gamma, Weibull-exponential, log logistic, exponential, generalized exponential, Rayleigh, generalized Rayleigh, generalized Pareto, linear exponential and Burr satisfy the characterizing differential equation defined in (1.3) or (1.4) with appropriate choices for the $p, q, a^{\prime} s, b^{\prime} s$ and $c$ as demonstrated in Remarks 2.2 and 2.3 in Section 2. Therefore, the recurrence relations for single and product moments of progressively Type-II right censored order statistics as well as those for the usual order statistics from the above mentioned doubly truncated distributions can easily be deduced from the results derived in Sections 2 and 3, as special cases.

Thus, the results presented in this paper will generalize and unify the earlier results in this direction for the moments of the usual order statistics as well as those for the progressively Type-II right censored order statistics due to several authors like Joshi (1978, 1982), Balakrishnan (1985), Balakrishnan and Joshi (1981, 1982, 1984), Balakrishnan and Malik (1986, 1987), Khan and Khan (1987), Saran and Pushkarna (1999 a, b, c; 2000 a, b; 2001; 2010), Aggarwala and Balakrishnan (1996), Saran and Pande (2012), Balakrishnan and Aggarwala (2000, Sections 4.4 and 4.5), etc.

\section{Recurrence relations for single moments}

In this section, we shall establish several recurrence relations for single moments of progressively Type-II right censored order statistics from a doubly truncated continuous distribution satisfying the characterizing differential equation (1.3) or, equivalently, (1.4).

Using (1.1), we have

$$
\begin{gathered}
\mu_{r: m: n}^{\left(R_{1}, R_{2}, \ldots, R_{m}\right)^{(k)}}=E\left[X_{r: m: n}^{\left(R_{1}, R_{2}, \ldots, R_{m}\right)}\right]^{k} \\
=A(n, m-1) \int_{Q_{1} \leq x_{1} \ll_{2}<\ldots<x_{m} \leq P_{1}} \int_{r} \ldots x_{t=1}^{k} \prod_{t}^{m} f\left(x_{t}\right)\left[1-F\left(x_{t}\right)\right]^{R_{t}} d x_{t} .
\end{gathered}
$$


Theorem 2.1. For $i \geq 0$,

$$
\sum_{t=0}^{p} a_{t} \mu_{1: 1: 1}^{(0)}{ }^{(i+t)}=\sum_{j=0}^{q} \frac{b_{j}}{i+j+1}\left(c P_{1}^{i+j+1}-d Q_{1}^{i+j+1}+\mu_{1: 1: 1}^{(0)}{ }^{(i+j+1)}\right) .
$$

Proof. From (2.1), for $n=m=r=1$, we obtain

$$
\sum_{t=0}^{p} a_{t} \mu_{1: 1: 1}^{(0)}{ }^{(i+t)}=\int_{Q_{1}}^{P_{1}} \sum_{t=0}^{p} a_{t} x_{1}^{i+t} f\left(x_{1}\right) d x_{1}
$$

Making use of (1.4), we get

$$
\begin{aligned}
\sum_{t=0}^{p} a_{t} \mu_{1: 1: 1}^{(0)}{ }^{(i+t)}=d \sum_{j=0}^{q} b_{j} & \int_{Q_{1}}^{P_{1}} x_{1}^{i+j} d x_{1}-\sum_{j=0}^{q} b_{j} \int_{Q_{1}}^{P_{1}} x_{1}^{i+j} F\left(x_{1}\right) d x_{1} \\
& =d \sum_{j=0}^{q} \frac{b_{j}}{i+j+1}\left(P_{1}^{i+j+1}-Q_{1}^{i+j+1}\right)-\sum_{j=0}^{q} \frac{b_{j}}{i+j+1}\left(P_{1}^{i+j+1}-\mu_{1: 1: 1}^{(0)}{ }^{(i+j+1)}\right),
\end{aligned}
$$

where the last term on the right hand side has been obtained by integration by parts. This on simplification leads to (2.2).

Theorem 2.2. For $n \geq 2$ and $i \geq 0$,

$$
\sum_{t=0}^{p} a_{t} \mu_{1: 1: n}^{(n-1)^{(i+t)}}=n \sum_{j=0}^{q} \frac{b_{j}}{i+j+1}\left[c \mu_{1: 1: n-1}^{(n-2)}{ }^{(i+j+1)}+\mu_{1: 1: n}^{(n-1)^{(i+j+1)}}-d Q_{1}{ }^{i+j+1}\right] .
$$

Proof. Proceeding in a similar manner as in Theorem 2.1, one can easily establish the relation in (2.3).

Theorem 2.3. For $2 \leq m \leq n-1, i \geq 0$ and $R_{1} \geq 1$,

$$
\begin{array}{r}
\sum_{t=0}^{p} a_{t} \mu_{1: m: n}^{\left(R_{1}, R_{2}, \ldots, R_{m}\right)^{(i+t)}}=\sum_{j=0}^{q} \frac{b_{j}}{i+j+1}\left[\frac { n c } { ( n - 1 ) } \left\{\left(n-R_{1}-1\right) \mu_{1: m-1: n-1}^{\left(R_{1}+R_{2}, R_{3}, \ldots, R_{m}\right)}{ }^{(i+j+1)}\right.\right. \\
\left.+R_{1} \mu_{1: m: n-1}^{\left(R_{1}-1, R_{2}, \ldots, R_{m}\right)}{ }^{(i+j+1)}\right\}-n d Q_{1}^{i+j+1} \\
\left.+\left(n-R_{1}-1\right) \mu_{1: m-1: n}^{\left(R_{1}+R_{2}+1, R_{3}, \ldots, R_{m}\right)^{(i+j+1)}}+\left(R_{1}+1\right) \mu_{1: m: n}^{\left(R_{1}, R_{2}, \ldots, R_{m}\right)}{ }^{(i+j+1)}\right]
\end{array}
$$

and, for $2 \leq m \leq n-1, i \geq 0$ and $R_{1}=0$,

$$
\begin{aligned}
\sum_{t=0}^{p} a_{t} \mu_{1: m: n}^{\left(0, R_{2}, \ldots, R_{m}\right)}{ }^{(i+t)} & =\sum_{j=0}^{q} \frac{b_{j}}{i+j+1}\left[n c \mu_{1: m-1: n-1}^{\left(R_{2}, R_{3}, \ldots, R_{m}\right)}\right)^{(i+j+1)}-n d Q_{1}^{i+j+1} \\
+ & \left.(n-1) \mu_{1: m-1: n}^{\left(R_{2}+1, R_{3}, \ldots, R_{m}\right)^{(i+j+1)}}+\mu_{1: m: n}^{\left(0, R_{2}, \ldots, R_{m}\right)}{ }^{(i+j+1)}\right] .
\end{aligned}
$$

Proof. The relations in (2.4) and (2.5) may be proved by following exactly the same steps as those used in proving Theorem 2.4, which is presented next. 
Theorem 2.4. For $2 \leq r \leq m-1, m \leq n-1, i \geq 0$ and $R_{r} \geq 1$,

$$
\begin{array}{r}
\left.\sum_{t=0}^{p} a_{t} \mu_{r: m: n}^{\left(R_{1}, R_{2}, \ldots, R_{m}\right)}\right)^{(i+t)}=\sum_{j=0}^{q} \frac{b_{j}}{i+j+1}\left[c \left\{\frac{A(n, r)}{A(n-1, r-1)} \mu_{r: m-1: n-1}^{\left(R_{1}, R_{2}, \ldots, R_{r-1}, R_{r}+R_{r+1}, R_{r+2}, \ldots, R_{m}\right)}{ }^{(i+j+1)}\right.\right. \\
-\frac{A(n, r-1)}{A(n-1, r-2)} \mu_{r-1: m-1: n-1}^{\left(R_{1}, R_{2}, \ldots, R_{r-2}, R_{r-1}+R_{r}, R_{r+1}, \ldots, R_{m}\right)}{ }^{(i+j+1)} \\
\left.+\frac{A(n, r-1)}{A(n-1, r-1)} R_{r} \mu_{r: m: n-1}^{\left(R_{1}, R_{2}, \ldots, R_{r-1}, R_{r}-1, R_{r+1}, \ldots, R_{m}\right)}{ }^{(i+j+1)}\right\} \\
+\left(n-S_{r}-r\right) \mu_{r: m-1: n}^{\left(R_{1}, R_{2}, \ldots, R_{r-1}, R_{r}+R_{r+1}+1, R_{r+2}, \ldots, R_{m}\right)}{ }^{(i+j+1)} \\
-\left(n-S_{r-1}-r+1\right) \mu_{r-1: m-1: n}^{\left(R_{1}, R_{2}, \ldots, R_{r-2}, R_{r-1}+R_{r}+1, R_{r+1}, \ldots, R_{m}\right)}{ }^{(i+j+1)} \\
\left.\left.+\left(R_{r}+1\right) \mu_{r: m}^{\left(R_{1}, R_{2}, \ldots, R_{m}\right)}\right)^{(i+j+1)}\right],
\end{array}
$$

and, for $2 \leq r \leq m-1, m \leq n, i \geq 0$ and $R_{r}=0$,

$$
\begin{array}{r}
\left.\sum_{t=0}^{p} a_{t} \mu_{r: m: n}^{\left(R_{1}, R_{2}, \ldots, R_{r-1}, 0, R_{r+1}, \ldots, R_{m}\right)}\right)^{(i+t)}=\sum_{j=0}^{q} \frac{b_{j}}{i+j+1}\left[c \left\{\frac{A(n, r)}{A(n-1, r-1)} \mu_{r: m-1: n-1}^{\left(R_{1}, R_{2}, \ldots, R_{r-1}, R_{r+1}, \ldots, R_{m}\right)}{ }^{(i+j+1)}\right.\right. \\
\left.-\frac{A(n, r-1)}{A(n-1, r-2)} \mu_{r-1: m-1: n-1}^{\left(R_{1}, R_{2}, \ldots, R_{r-1}, R_{r+1}, \ldots, R_{m}\right)}{ }^{(i+j+1)}\right\} \\
+\left(n-S_{r-1}-r\right) \mu_{r: m-1: n}^{\left(R_{1}, R_{2}, \ldots, R_{r-1}, R_{r+1}+1, R_{r+2}, \ldots, R_{m}\right)}{ }^{(i+j+1)} \\
-\left(n-S_{r-1}-r+1\right) \mu_{r-1: m-1: n}^{\left(R_{1}, R_{2}, \ldots, R_{r-2}, R_{r-1}+1, R_{r+1}, \ldots, R_{m}\right)^{(i+j+1)}} \\
\left.+\mu_{r: m: n}^{\left(R_{1}, R_{2}, \ldots, R_{r-1}, 0, R_{r+1}, \ldots, R_{m}\right)}{ }^{(i+j+1)}\right],
\end{array}
$$

where $S_{i}=R_{1}+R_{2}+\ldots+R_{i}, \quad 1 \leq i \leq m$.

Proof. In order to establish (2.6), we note on using (2.1) that

$$
\begin{gathered}
\sum_{t=0}^{p} a_{t} \mu_{r: m: n}^{\left(R_{1}, R_{2}, \ldots, R_{m}\right)}{ }^{(i+t)}=A(n, m-1) \int_{Q_{1} \leq x_{1}<\ldots<x_{r-1}<x_{r+1}<\ldots<x_{m} \leq P_{1}} I\left(x_{r-1}, x_{r+1}\right) \\
\cdot \prod_{u=1, u \neq r}^{m}\left[1-F\left(x_{u}\right)\right]^{R_{u}} f\left(x_{u}\right) d x_{u},
\end{gathered}
$$

where

$$
I\left(x_{r-1}, x_{r+1}\right)=\int_{x_{r-1}}^{x_{r+1}} \sum_{t=0}^{p} a_{t} x_{r}^{i+t}\left[1-F\left(x_{r}\right)\right]^{R_{r}} f\left(x_{r}\right) d x_{r} .
$$

Making use of the relation in (1.3) and splitting the integral accordingly into two, we have

$$
I\left(x_{r-1}, x_{r+1}\right)=c \sum_{j=0}^{q} b_{j} I_{0}\left(x_{r-1}, x_{r+1}\right)+\sum_{j=0}^{q} b_{j} I_{1}\left(x_{r-1}, x_{r+1}\right),
$$


where

$$
I_{a}\left(x_{r-1}, x_{r+1}\right)=\int_{x_{r-1}}^{x_{r+1}} x_{r}^{i+j}\left[1-F\left(x_{r}\right)\right]^{R_{r}+a} d x_{r}, \quad a=0,1 .
$$

Integration by parts yields,

$$
\begin{array}{r}
I_{a}\left(x_{r-1}, x_{r+1}\right)=\frac{1}{i+j+1}\left[x_{r+1}^{i+j+1}\left[1-F\left(x_{r+1}\right)\right]^{R_{r}+a}-x_{r-1}^{i+j+1}\left[1-F\left(x_{r-1}\right)\right]^{R_{r}+a}\right. \\
\left.+\left(R_{r}+a\right) \int_{x_{r-1}}^{x_{r+1}} x_{r}^{i+j+1}\left[1-F\left(x_{r}\right)\right]^{R_{r}+a-1} f\left(x_{r}\right) d x_{r}\right] .
\end{array}
$$

Upon substituting for $I_{0}\left(x_{r-1}, x_{r+1}\right)$ and $I_{1}\left(x_{r-1}, x_{r+1}\right)$ from (2.11) in (2.10) and then substituting the resultant expression for $I\left(x_{r-1}, x_{r+1}\right)$ in (2.8) and simplifying, it leads to (2.6).

To derive (2.7), we proceed in exactly the same way as in the proof of (2.6) given above. Other things remain the same as in the proof of (2.6), the only change will be in the value of $I_{a}\left(x_{r-1}, x_{r+1}\right), a=0,1$, which for the case $R_{r}=0$ comes out to be

$$
I_{0}\left(x_{r-1}, x_{r+1}\right)=\frac{1}{i+j+1}\left[x_{r+1}^{i+j+1}-x_{r-1}^{i+j+1}\right]
$$

and

$$
I_{1}\left(x_{r-1}, x_{r+1}\right)=\frac{1}{i+j+1}\left[x_{r+1}^{i+j+1}\left(1-F\left(x_{r+1}\right)\right)-x_{r-1}^{i+j+1}\left(1-F\left(x_{r-1}\right)\right)+\int_{x_{r-1}}^{x_{r+1}} x_{r}^{i+j+1} f\left(x_{r}\right) d x_{r}\right] .
$$

Thus, in the case $R_{r}=0$, (2.7) follows from (2.8), (2.10), (2.12) and (2.13).

Likewise, the recurrence relations given in the following theorem can also be established.

Theorem 2.5. For $2 \leq m \leq n-1, i \geq 0$ and $R_{m} \geq 1$,

$$
\begin{array}{r}
\left.\sum_{t=0}^{p} a_{t} \mu_{m: m: n}^{\left(R_{1}, R_{2}, \ldots, R_{m}\right)}\right)^{(i+t)}=\sum_{j=0}^{q} \frac{b_{j}}{i+j+1}\left[c \left\{\frac{A(n, m-1)}{A(n-1, m-1)} R_{m} \mu_{m: m: n-1}^{\left(R_{1}, R_{2}, \ldots, R_{m-1}, R_{m}-1\right)}{ }^{(i+j+1)}\right.\right. \\
\left.-\frac{A(n, m-1)}{A(n-1, m-2)} \mu_{m-1: m-1: n-1}^{\left(R_{1}, R_{2}, \ldots, R_{m-2}, R_{m-1}+R_{m}\right)}{ }^{(i+j+1)}\right\} \\
-\left(n-S_{m-1}-m+1\right) \mu_{m-1: m-1: n}^{\left(R_{1}, R_{2}, \ldots, R_{m-2}, R_{m-1}+R_{m}+1\right)}{ }^{(i+j+1)} \\
\left.+\left(R_{m}+1\right) \mu_{m: m: n}^{\left(R_{1}, R_{2}, \ldots, R_{m}\right)}{ }^{(i+j+1)}\right],
\end{array}
$$


and, for $2 \leq m \leq n, i \geq 0$ and $R_{m}=0$,

$$
\begin{aligned}
\left.\sum_{t=0}^{p} a_{t} \mu_{m: m: n}^{\left(R_{1}, R_{2}, \ldots, R_{m-1}, 0\right)}\right)^{(i+t)} & =\sum_{j=0}^{q} \frac{b_{j}}{i+j+1}\left[c \left\{\frac{A(n, m-1)}{A(n-1, m-2)} P_{1}^{i+j+1}\right.\right. \\
& \left.-\frac{A(n, m-1)}{A(n-1, m-2)} \mu_{m-1: m-1: n-1}^{\left(R_{1}, R_{2}, \ldots, R_{m-1}\right)(i+j+1)}\right\} \\
& -\left(n-S_{m-1}-m+1\right) \mu_{m-1: m-1: n}^{\left(R_{1}, R_{2}, \ldots, R_{m-2}, R_{m-1}+1\right)}(i+j+1) \\
& \left.+\mu_{m: m: n}^{\left(R_{1}, R_{2}, \ldots, R_{m-1}, 0\right)}{ }^{(i+j+1)}\right] .
\end{aligned}
$$

Remark 2.1. It may be mentioned that if $R_{1}=R_{2}=\ldots=R_{k-1}=0$, i.e., there is no censoring before the time of the $k^{\text {th }}$ failure, then the first $k$ progressively Type-II right censored order statistics are simply the first $k$ usual order statistics. Thus, for the special case $R_{1}=R_{2}=\ldots=R_{m}=0$, so that $m=n$ in which case the progressively censored order statistics become the usual order statistics $X_{1: n}, X_{2: n}, \ldots, X_{n: n}$, the recurrence relations established in Section 2 would reduce to the corresponding recurrence relations for the single moments of usual order statistics from the general class of doubly truncated distributions satisfying the characterizing differential equation (1.3) or (1.4), thus verifying the results of Saran and Pushkarna (2010).

Remark 2.2. Setting

$$
\begin{gathered}
a_{i}= \begin{cases}1 ; & \text { when } i=0 \\
0 ; & \text { when } 1 \leq i \leq p-1 \\
\theta ; & \text { when } i=p,\end{cases} \\
b_{j}= \begin{cases}v p \theta ; & \text { when } j=p-1 \\
0 & ; \text { when } j \neq p-1,\end{cases}
\end{gathered}
$$

and

$$
c=(1-P) /(P-Q)=P_{2} \text {, say }\left(\text { i.e., } d=1+P_{2}=Q_{2} \text {, say }\right),
$$

we observe that (1.4), for $q \geq p-1$, reduces to

$$
\left(1+\theta x^{p}\right) f(x)=v p \theta x^{p-1}\left(Q_{2}-F(x)\right),
$$

which is the characterizing differential equation for the doubly truncated Burr type XII distribution (cf. Khan and Khan (1987) and Saran and Pushkarna (2001)) with p.d.f. in the form

$$
f(x)=\frac{v p \theta x^{p-1}\left(1+\theta x^{p}\right)^{-(v+1)}}{(P-Q)}, Q_{1} \leq x \leq P_{1}, v>0, p>0, \theta>0 .
$$

For the above values of $a_{i}, b_{j}, c$ and $d$, as given in (2.16), the recurrence relations in Section 2 will reduce to and verify the corresponding recurrence relations established by Saran and Pushkarna 
(2001) for the progressively Type-II right censored order statistics from doubly truncated Burr type XII distribution.

It may be mentioned that one can derive similar recurrence relations for doubly truncated Lomax, Weibull, Weibull-gamma, Weibull-exponential, log logistic, exponential, generalized exponential, Rayleigh, generalized Rayleigh and generalized Pareto distributions, since these distributions are some versions of Burr type XII distribution by taking different values of the parameters involved as discussed in Tadikamalla (1980) and Saran and Pushkarna (2001).

Remark 2.3. Setting

$$
\begin{aligned}
& a_{i}= \begin{cases}1, & \text { when } i=0 \\
0, & \text { when } i \neq 0,\end{cases} \\
& b_{j}= \begin{cases}\lambda, & \text { when } j=0 \\
v, & \text { when } j=1 \\
0, & \text { when } \mathrm{j} \geq 2,\end{cases}
\end{aligned}
$$

and

$$
c=(1-P) /(P-Q)=P_{2}, \operatorname{say}\left(i . e ., d=1+P_{2}=Q_{2}, \text { say }\right),
$$

we observe that (1.3), or equivalently (1.4), reduces to the well-known characterizing differential equation for the doubly truncated linear exponential distribution (cf. Saran and Pushkarna (1999b) and also the recurrence relations given in Section 2 reduce to the corresponding results for progressively Type-II right censored order statistics from doubly truncated linear exponential distribution.

\section{Recurrence relations for product moments}

Using (1.1) we can write the product moments of the progressively Type-II right censored order statistics as follows:

$$
\begin{aligned}
& \mu_{r, s: m: n}^{\left(R_{1}, R_{2}, \ldots, R_{m}\right)^{\left(k_{1}, k_{2}\right)}}=E\left[\left\{X_{r: m: n}^{\left(R_{1}, R_{2}, \ldots, R_{m}\right)}\right\}^{k_{1}}\left\{X_{s: m: n}^{\left(R_{1}, R_{2}, \ldots, R_{m}\right)}\right\}^{k_{2}}\right] \\
& =A(n, m-1) \int_{Q_{1} \leq x_{1}<x_{2}<\ldots<x_{m} \leq P_{1}} \int_{r} \ldots x_{r}^{k_{1}} x_{s}^{k_{2}} \prod_{t=1}^{m} f\left(x_{t}\right)\left[1-F\left(x_{t}\right)\right]^{R_{t}} d x_{t},
\end{aligned}
$$

where $1 \leq r<s \leq m \leq n$ and $k_{1}, k_{2} \geq 0$. Also

$$
\begin{aligned}
& \mu_{r, r: m: n}^{\left(R_{1}, R_{2}, \ldots, R_{m}\right)^{\left(k_{1}, k_{2}\right)}}=E\left[\left\{X_{r: m: n}^{\left(R_{1}, R_{2}, \ldots, R_{m}\right)}\right\}^{k_{1}}\left\{X_{r: m: n}^{\left(R_{1}, R_{2}, \ldots, R_{m}\right)}\right\}^{k_{2}}\right] \\
& =\mu_{r: m: n}^{\left(R_{1}, R_{2}, \ldots, R_{m}\right)^{\left(k_{1}+k_{2}\right)},}
\end{aligned}
$$

as defined in (2.1), where $1 \leq r \leq m \leq n$ and $k_{1}, k_{2} \geq 0$.

In this section, we shall derive various recurrence relations for the product moments of progressively Type-II right censored order statistics from a doubly truncated continuous distribution with 
p.d.f. $f(x)$ and c.d.f. $F(x)$ satisfying the characterizing differential equation (1.3) or (1.4).

Theorem 3.1. For $2 \leq s \leq m \leq n-R_{1}, i, k \geq 0$ and $R_{1} \geq 1$,

$$
\begin{aligned}
& \sum_{t=0}^{p} a_{t} \mu_{1, s: m: n}^{\left(R_{1}, R_{2}, \ldots, R_{m}\right)}{ }^{(i+t, k)}=\sum_{j=0}^{q} \frac{b_{j}}{i+j+1}\left[\frac { n c } { n - 1 } \left\{\left(n-R_{1}-1\right) \mu_{1, s-1: m-1: n-1}^{\left(R_{1}+R_{2}, R_{3}, \ldots, R_{m}\right)}{ }^{(i+j+1, k)}\right.\right. \\
& \left.+R_{1} \mu_{1, s: m: n-1}^{\left(R_{1}-1, R_{2}, R_{3}, \ldots, R_{m}\right)^{(i+j+1, k)}}\right\}-n d Q_{1}^{i+j+1} \mu_{s-1: m-1: n-R_{1}-1}^{\left(R_{2}, R_{3}, \ldots, R_{m}\right)^{(k)}} \\
& \left.+\left(n-R_{1}-1\right) \mu_{1, s-1: m-1: n}^{\left(R_{1}+R_{2}+1, R_{3}, R_{4}, \ldots, R_{m}\right)^{(i+j+1, k)}}+\left(R_{1}+1\right) \mu_{1, s: m: n}^{\left(R_{1}, R_{2}, \ldots, R_{m}\right)}{ }^{(i+j+1, k)}\right],
\end{aligned}
$$

and, for $2 \leq s \leq m \leq n, i, k \geq 0$ and $R_{1}=0$,

$$
\begin{array}{r}
\sum_{t=0}^{p} a_{t} \mu_{1, s: m: n}^{\left(0, R_{2}, \ldots, R_{m}\right)}{ }^{(i+t, k)}=\sum_{j=0}^{q} \frac{b_{j}}{i+j+1}\left[n c \mu_{1, s-1: m-1: n-1}^{\left(R_{2}, R_{3}, \ldots, R_{m}\right)}{ }^{(i+j+1, k)}-n d Q_{1}^{i+j+1} \mu_{s-1: m-1: n-1}^{\left(R_{2}, R_{3}, \ldots, R_{m}\right)^{(k)}}\right. \\
\left.+(n-1) \mu_{1, s-1: m-1: n}^{\left(R_{2}+1, R_{3}, \ldots, R_{m}\right)}{ }^{(i+j+1, k)}+\mu_{1, s: m: n}^{\left(0, R_{2}, R_{3}, \ldots, R_{m}\right)}{ }^{(i+j+1, k)}\right] .
\end{array}
$$

Proof. The relations in (3.3) and (3.4) may be proved by following exactly the same steps as those used in proving Theorem 3.2, which is presented next to Remark 3.1.

Remark 3.1. It may be remarked that for the case $s=2$, Theorem 3.1 remains valid provided we replace $\mu_{1,1: m: n}^{\left(R_{1}, R_{2}, \ldots, R_{m}\right)^{(i, k)}}$ by $\mu_{1: m: n}^{\left(R_{1}, R_{2}, \ldots, R_{m}\right)}{ }^{(i+k)}$, as mentioned in (3.2).

Theorem 3.2. For $2 \leq r<s \leq m<n, i, k \geq 0$ and $R_{r} \geq 1$,

$$
\begin{aligned}
& \sum_{t=0}^{p} a_{t} \mu_{r, s: m: n}^{\left(R_{1}, R_{2}, \ldots, R_{m}\right)}{ }^{(i+t, k)}=\sum_{j=0}^{q} \frac{b_{j}}{i+j+1}\left[c\left\{\frac{A(n, r)}{A(n-1, r-1)} \mu_{r, s-1: m-1: n-1}^{\left(R_{1}, R_{2}, \ldots, R_{r-1}, R_{r}+R_{r+1}, R_{r+2}, \ldots, R_{m}\right)}\right)^{(i+j+1, k)}\right. \\
& -\frac{A(n, r-1)}{A(n-1, r-2)} \mu_{r-1, s-1: m-1: n-1}^{\left(R_{1}, R_{2}, \ldots, R_{r-2}, R_{r-1}+R_{r}, R_{r+1}, \ldots, R_{m}\right)}{ }^{(i+j+1, k)} \\
& \left.+R_{r} \frac{A(n, r-1)}{A(n-1, r-1)} \mu_{r, s: m: n-1}^{\left(R_{1}, R_{2}, \ldots, R_{r-1}, R_{r}-1, R_{r+1}, \ldots, R_{m}\right)}{ }^{(i+j+1, k)}\right\} \\
& +\left(n-S_{r}-r\right) \mu_{r, s-1: m-1: n}^{\left(R_{1}, R_{2}, \ldots, R_{r-1}, R_{r}+R_{r+1}+1, R_{r+2}, \ldots, R_{m}\right)}(i+j+1, k) \\
& -\left(n-S_{r-1}-r+1\right) \mu_{r-1, s-1: m-1: n}^{\left(R_{1}, R_{2}, \ldots, R_{r-2}, R_{r-1}+R_{r}+1, R_{r+1}, \ldots, R_{m}\right)^{(i+j+1, k)}} \\
& \left.\left.+\left(R_{r}+1\right) \mu_{r, s: m: n}^{\left(R_{1}, R_{2}, \ldots, R_{m}\right)}\right)^{(i+j+1, k)}\right] .
\end{aligned}
$$

Proof. From (3.1), let us consider for $2 \leq r<s \leq m<n, i, k \geq 0$ and $R_{r} \geq 1$,

$$
\begin{array}{r}
\sum_{t=0}^{p} a_{t} \mu_{r, s: m: n}^{\left(R_{1}, R_{2}, \ldots, R_{m}\right)^{(i+t, k)}}=A(n, m-1) \int_{Q_{1} \leq x_{1}<\ldots<x_{r-1}<x_{r+1}<\ldots<x_{m} \leq P_{1}} x_{s}^{k} I\left(x_{r-1}, x_{r+1}\right) \\
\cdot \prod_{u=1, u \neq r}^{m} f\left(x_{u}\right)\left[1-F\left(x_{u}\right)\right]^{R_{u}} d x_{u},
\end{array}
$$


where $I\left(x_{r-1}, x_{r+1}\right)$ is the same as given in equation (2.9), or equivalently, in equations (2.10) and (2.11). Now upon using (2.11) in (2.10) and then putting the value of $I\left(x_{r-1}, x_{r+1}\right)$, so obtained, into the equation (3.6) and then simplifying, it leads to (3.5).

Theorem 3.3. For $2 \leq r<s \leq m<n, i, k \geq 0$ and $R_{r}=0$,

$$
\begin{aligned}
\sum_{t=0}^{p} a_{t} \mu_{r, s: m: n}^{\left(R_{1}, R_{2}, \ldots, R_{r-1}, 0, R_{r+1}, \ldots, R_{m}\right)}{ }^{(i+t, k)} & \\
& =\sum_{j=0}^{q} \frac{b_{j}}{i+j+1}\left[c \left\{\frac{A(n, r)}{A(n-1, r-1)} \mu_{r, s-1: m-1: n-1}^{\left(R_{1}, R_{2}, \ldots, R_{r-1}, R_{r+1}, \ldots, R_{m}\right)}(i+j+1, k)\right.\right. \\
& \left.-\frac{A(n, r-1)}{A(n-1, r-2)} \mu_{r-1, s-1: m-1: n-1}^{\left(R_{1}, R_{2}, \ldots, R_{r-1}, R_{r+1}, \ldots, R_{m}\right)^{(i+j+1, k)}}\right\} \\
& \left.+\left(n-S_{r-1}-r\right) \mu_{r, s-1: m-1: n}^{\left(R_{1}, R_{2}, \ldots, R_{r-1}, R_{r+1}+1, R_{r+2}, \ldots, R_{m}\right)}\right)^{(i+j+1, k)} \\
& -\left(n-S_{r-1}-r+1\right) \mu_{r-1, s-1: m-1: n}^{\left(R_{1}, R_{2}, \ldots, R_{r-2}, R_{r-1}+1, R_{r+1}, \ldots, R_{m}\right)}(i+j+1, k) \\
& +\mu_{r, s: m: n}^{\left(R_{1}, R_{2}, \ldots, R_{r-1}, 0, R_{r+1}, \ldots, R_{m}\right)}(i+j+1, k)
\end{aligned}
$$

Proof. The relation in (3.7) may be proved by following the similar steps as those used in proving (3.5).

Remark 3.2. It may be noted that Theorems 3.2 and 3.3 hold even for $s=r+1$ without altering the proof, provided we realize that $\left.\mu_{r, r: m: n}^{\left(R_{1}, R_{2}, \ldots, R_{m}\right)}\right)^{(i, k)}=\mu_{r: m: n}^{\left(R_{1}, R_{2}, \ldots, R_{m}\right)}{ }^{(i+k)}$, as mentioned in (3.2).

Likewise, the recurrence relations given in the following theorems can also be established.

Theorem 3.4. For $1 \leq r<s<m<n, i, k \geq 0$ and $R_{s} \geq 1$,

$$
\begin{aligned}
& \sum_{t=0}^{p} a_{t} \mu_{r, s: m: n}^{\left(R_{1}, R_{2}, \ldots, R_{m}\right)}{ }^{(i, k+t)}=\sum_{j=0}^{q} \frac{b_{j}}{j+k+1}\left[c \left\{\frac{A(n, s)}{A(n-1, s-1)} \mu_{r, s: m-1: n-1}^{\left(R_{1}, R_{2}, \ldots, R_{s-1}, R_{s}+R_{s+1}, R_{s+2}, \ldots, R_{m}\right)}{ }^{(i, j+k+1)}\right.\right. \\
& -\frac{A(n, s-1)}{A(n-1, s-2)} \mu_{r, s-1: m-1: n-1}^{\left(R_{1}, R_{2}, \ldots, R_{s-2}, R_{s-1}+R_{s}, R_{s+1}, \ldots, R_{m}\right)}{ }^{(i, j+k+1)} \\
& \left.+R_{s} \frac{A(n, s-1)}{A(n-1, s-1)} \mu_{r, s: m: n-1}^{\left(R_{1}, R_{2}, \ldots, R_{s-1}, R_{s}-1, R_{s+1}, \ldots, R_{m}\right)}{ }^{(i, j+k+1)}\right\} \\
& +\left(n-S_{s}-s\right) \mu_{r, s: m-1: n}^{\left(R_{1}, R_{2}, \ldots, R_{s-1}, R_{s}+R_{s+1}+1, R_{s+2}, \ldots, R_{m}\right)}{ }^{(i, j+k+1)} \\
& -\left(n-S_{s-1}-s+1\right) \mu_{r, s-1: m-1: n}^{\left(R_{1}, R_{2}, \ldots, R_{s-2}, R_{s-1}+R_{s}+1, R_{s+1}, \ldots, R_{m}\right)^{(i, j+k+1)}}
\end{aligned}
$$

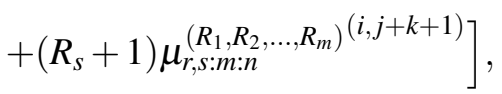


and, for $1 \leq r<s<m \leq n, i, k \geq 0$ and $R_{s}=0$,

$$
\begin{aligned}
& \sum_{t=0}^{p} a_{t} \mu_{r, s: m: n}^{\left(R_{1}, R_{2}, \ldots, R_{s-1}, 0, R_{s+1}, \ldots, R_{m}\right)^{(i, j+k+1)}} \\
& =\sum_{j=0}^{q} \frac{b_{j}}{j+k+1}\left[c \left\{\frac{A(n, s)}{A(n-1, s-1)} \mu_{r, s: m-1: n-1}^{\left(R_{1}, R_{2}, \ldots, R_{s-1}, R_{s+1}, R_{s+2}, \ldots, R_{m}\right)}{ }^{(i, j+k+1)}\right.\right. \\
& \left.-\frac{A(n, s-1)}{A(n-1, s-2)} \mu_{r, s-1: m-1: n-1}^{\left(R_{1}, R_{2}, \ldots, R_{s-2}, R_{s-1}, R_{s+1}, \ldots, R_{m}\right)^{(i, j+k+1)}}\right\} \\
& +\left(n-S_{s}-s\right) \mu_{r, s: m-1: n}^{\left(R_{1}, R_{2}, \ldots, R_{s-1}, R_{s+1}+1, R_{s+2}, \ldots, R_{m}\right)}{ }^{(i, j+k+1)} \\
& -\left(n-S_{s-1}-s+1\right) \mu_{r, s-1: m-1: n}^{\left(R_{1}, R_{2}, \ldots, R_{s-2}, R_{s-1}+1, R_{s+1}, \ldots, R_{m}\right)}(i, j+k+1) \\
& \left.\left.+\mu_{r, s: m: n}^{\left(R_{1}, R_{2}, \ldots, R_{s-1}, 0, R_{s+1}, \ldots, R_{m}\right)}\right)^{(i, j+k+1)}\right] .
\end{aligned}
$$

Theorem 3.5. For $1 \leq r<m<n, i, k \geq 0$ and $R_{m} \geq 1$,

$$
\begin{array}{r}
\left.\sum_{t=0}^{p} a_{t} \mu_{r, m: m: n}^{\left(R_{1}, R_{2}, \ldots, R_{m}\right)}\right)^{(i, k+t)}=\sum_{j=0}^{q} \frac{b_{j}}{j+k+1}\left[c\left\{\frac{A(n, m-1)}{A(n-1, m-1)} R_{m} \mu_{r, m: m: n-1}^{\left(R_{1}, R_{2}, \ldots, R_{m-1}, R_{m}-1\right)}\right)^{(i, j+k+1)}\right. \\
\left.\left.-\frac{A(n, m-1)}{A(n-1, m-2)} \mu_{r, m-1: m-1: n-1}^{\left(R_{1}, R_{2}, \ldots, R_{m-2}, R_{m-1}+R_{m}\right)}\right)^{(i, j+k+1)}\right\} \\
\left.-\left(n-S_{m-1}-m+1\right) \mu_{r, m-1: m-1: n}^{\left(R_{1}, R_{2}, \ldots, R_{m-2}, R_{m-1}+R_{m}+1\right)}\right)^{(i, j+k+1)} \\
\left.\left.+\left(R_{m}+1\right) \mu_{r, m: m: n}^{\left(R_{1}, R_{2}, \ldots, R_{m}\right)}\right)^{(i, j+k+1)}\right],
\end{array}
$$

and, for $1 \leq r<m<n, i, k \geq 0$ and $R_{m}=0$,

$$
\begin{aligned}
\left.\sum_{t=0}^{p} a_{t} \mu_{r, m: m: n}^{\left(R_{1}, R_{2}, \ldots, R_{m-1}, 0\right)}\right)^{(i, k+t)} & =\sum_{j=0}^{q} \frac{b_{j}}{j+k+1}\left[c \frac { A ( n , m - 1 ) } { A ( n - 1 , m - 2 ) } \left\{P_{1}^{j+k+1} \mu_{r: m-1: n-1}^{\left(R_{1}, R_{2}, \ldots, R_{m-1}\right)^{(i)}}\right.\right. \\
& -\mu_{r, m-1: m-1: n-1}^{\left(R_{1}, R_{2}, \ldots, R_{m-1}\right)}(i, j+k+1) \\
& -\left(n-S_{m-1}-m+1\right) \mu_{r, m-1: m-1: n}^{\left(R_{1}, R_{2}, \ldots, R_{m-2}, R_{m-1}+1\right)^{(i, j+k+1)}} \\
& \left.+\mu_{r, m: m: n}^{\left(R_{1}, R_{2}, \ldots, R_{m-1}, 0\right)}{ }^{(i, j+k+1)}\right] .
\end{aligned}
$$

Remark 3.3. For the special case $R_{1}=R_{2}=\ldots=R_{m}=0$, the recurrence relations established in Section 3 reduce to the corresponding recurrence relations for product moments of usual order statistics from the general class of doubly truncated distributions (1.3), thus verifying the results of Saran and Pushkarna (2010).

Remark 3.4. For special values of $a_{i}, b_{j}, c$ and $d$, as given in (2.16), the recurrence relations in Section 3 will reduce to the corresponding recurrence relations for the product moments of progressively Type-II right censored order statistics from doubly truncated Burr type XII distribution, thus verifying the results established by Saran and Pushkarna (2001).

As pointed out earlier in Remarks 2.2 and 2.3, the recurrence relations for product moments of progressively Type-II right censored order statistics from doubly truncated Lomax, 
Weibull,Weibull-gamma, Weibull-exponential, log logistic, exponential, generalized exponential, Rayleigh, generalized Rayleigh, generalized Pareto and linear exponential distributions can be easily deduced from the results of Section 3 as special cases.

\section{Acknowledgements}

The authors are grateful to the two anonymous referees for giving valuable comments.

\section{References}

[1] R. Aggarwala, Progressive Censoring: A Review, in Handbook of Statistics, Vol. 20, Advances in Reliability, eds. N. Balakrishnan, and C.R. Rao (North-Holland, Amsterdam, The Netherlands, 2001), pp. 373-429.

[2] R. Aggarwala and N. Balakrishnan, Recurrence relations for single and product moments of progressive Type-II right censored order statistics from exponential and truncated exponential distributions, Ann. Inst. Statist. Math., 48(4) (1996) 757-771.

[3] N. Balakrishnan, Order statistics from the half logistic distribution, J. Statist. Comp. Simul., 20 (1985) 287-309.

[4] N. Balakrishnan and R. Aggarwala, Progressive censoring: Theory, Methods and Applications, (Birkhauser, Boston , 2000)

[5] N. Balakrishnan and P.C. Joshi, Moments of order statistics from doubly truncated power function distribution, Aligarh J. Statist. 1 (1981) 98-105.

[6] N. Balakrishnan and P.C. Joshi, Moments of order statistics from doubly truncated Pareto distribution, J. Indian Statist. Assoc. 20 (1982) 109-117.

[7] N. Balakrishnan and P.C. Joshi, Product moments of order statistics from doubly truncated exponential distribution, Naval Res. Logist. Quart. 31 (1984) 27-31.

[8] N. Balakrishnan and H.J. Malik, Order statistics from the linear-exponential distribution, Part I: Increasing hazard rate case, Commun. Statist.-Theor. Meth. 15(1) (1986) 179-203.

[9] N. Balakrishnan and H.J. Malik, Moments of order statistics from truncated log-logistic distribution, $J$. Statist. Plann. Infer. 17(2) (1987) 251-267.

[10] N. Balakrishnan and R.A. Sandhu, A simple simulational algorithm for generating progressive type-II censored samples, Amer. Statist. 49 (1995) 229-230.

[11] A.C. Cohen, Progressively censored samples in life testing, Technometrics 5 (1963) 327-329.

[12] P.C. Joshi, Recurrence relations between moments of order statistics from exponential and truncated exponential distributions, Sankhya B 39 (1978) 362-371.

[13] P.C. Joshi, A note on the mixed moments of order statistics from exponential and truncated exponential distributions, J. Statist. Plann. Infer. 6 (1982) 13-16.

[14] A.H. Khan and I.A. Khan, Moments of order statistics from Burr distribution and its characterizations, Metron XLV, (1-2) (1987) 21-29.

[15] J. Saran and V. Pande, Recurrence relations for moments of progressively Type-II right censored order statistics from half logistic distribution, J. Statist. Theory and Applications 11(1) (2012) 87-96.

[16] J. Saran and N. Pushkarna, Moments of order statistics from doubly truncated Lomax distribution, $J$. Statist. Res. 33(1) (1999a) 57-66.

[17] J. Saran and N. Pushkarna, Moments of order statistics from doubly truncated linear-exponential distribution, J. Korean Statist. Soc. 28(3) (1999b) 279-296.

[18] J. Saran and N. Pushkarna, Moments of order statistics from doubly truncated generalized exponential distribution, Aligarh J. Statist. 19 (1999c) 77-82.

[19] J. Saran and N. Pushkarna, Relationships for moments of order statistics from a generalized exponential distribution, Statistica 60(3) (2000a) 585-595.

[20] J. Saran and N. Pushkarna, Recurrence relations for moments of order statistics form doubly truncated generalized Rayleigh distribution, J. Statist. studies 20 (2000b) 27-31.

[21] J. Saran and N. Pushkarna, Recurrence relations for moments of progressive Type-II right censored order statistics from Burr distribution, Statistics 35 (2001) 495-507. 
Moments of Progressive Type-II...

[22] J. Saran and N. Pushkarna, Moments of order statistics from a general class of doubly truncated continuous distributions, Pak. J. Statist. 26(2) (2010) 327-337.

[23] P.K. Sen, Progressive censoring schemes, in Encyclopedia of Statistical Sciences, Vol. 7, eds. S. Kotz and N.L. Johnson (Wiley, New York, 1986), pp. 296-299.

[24] P.R. Tadikamalla, A look at the Burr and related distributions, International Statistical Review 48 (1980) 337-344. 